\title{
Implementation of a Massively Parallel Dynamic Security Assessment Platform for Large-Scale Grids
}

\author{
Ioannis Konstantelos, Member, IEEE, Geoffroy Jamgotchian, Simon H. Tindemans, Member, IEEE, \\ Philippe Duchesne, Stijn Cole, Christian Merckx, Goran Strbac, Member, IEEE, \\ and Patrick Panciatici, Senior Member, IEEE
}

\begin{abstract}
This paper presents a computational platform for dynamic security assessment (DSA) of large electricity grids, developed as part of the iTesla project. It leverages High Performance Computing (HPC) to analyze large power systems, with many scenarios and possible contingencies, thus paving the way for pan-European operational stability analysis. The results of the DSA are summarized by decision trees of 11 stability indicators. The platform's workflow and parallel implementation architecture is described in detail, including the way commercial tools are integrated into a plug-in architecture. A case study of the French grid is presented, with over 8000 scenarios and 1980 contingencies. Performance data of the case study (using 10,000 parallel cores) is analyzed, including task timings and data flows. Finally, the generated decision trees are compared with test data to quantify the functional performance of the DSA platform.
\end{abstract}

Index Terms-High performance computing, Monte Carlo methods, power system simulation, power system stability.

\section{INTRODUCTION}

$\mathrm{T}$ HE reliable operation of the electricity system is a task of paramount importance worldwide. In many cases, Transmission System Operators (TSOs) carry out ad hoc timedomain simulations for specific faults to ensure power system resilience against dynamic phenomena. Such simulations can be run hours ahead or close to real time, making use of forecasts as well as operators' experience to heuristically determine potential critical states and the corresponding control action to be taken.

Despite serving the industry well in the past, the existing operational doctrines are being challenged by three increasingly relevant factors. Firstly, the growing penetration of intermittent energy sources vastly expands the uncertainty state-space. As a result, even small prediction errors may result in an unrecoverable system state [1]. Secondly, the widespread deployment of power electronics devices across the network enable system operation closer to the stability limit while potentially leading to a growing range of disruptive dynamic phenomena. Finally, the increasing interconnectedness that characterizes modern electricity systems is likely to lead to wide-area stability issues, necessitating comprehensive analysis across multiple jurisdictions. In Europe, there have already

This research has received funding from the European Union $7^{\text {th }}$ Framework Programme under agreement $\mathrm{n}^{\circ}$ 283012. www.itesla-project.eu. I. Konstantelos, S. Tindemans and G. Strbac are with Imperial College London, been examples of this new reality, resulting in loss of load due to inter-area oscillations between countries [2].

Assessing the dynamic stability of a very large electrical system across a vast number of potential operating points and against a large set of potential faults is an exceptionally complex task which cannot be tackled analytically. Simulationbased Monte Carlo methods can be used instead to probe the system's post-fault behavior and identify the system's stability boundary. However, given that power system optimization and simulation are highly computationally-intensive tasks, such schemes entail a huge computational load.

Recent progress in accessible High Performance Computing (HPC) has the potential to bring complex analyses within practical reach [17]. Complex problems such as integrated system planning, power market simulation and optimization of operational decisions are starting to take full advantage of the transformative capabilities of HPC [8]. Application examples range from parallelized state estimation [19] to long-term system adequacy studies [18]. Industrial deployment of such applications is also progressing rapidly. For example, ISO New England is currently utilizing HPC to carry out securityconstrained unit commitment using robust optimization techniques, resulting in significant operational benefits [9].

One of the application areas gathering significant attention from researchers and industry is Dynamic Security Assessment (DSA), due to the contingency parallelization potential. DSA, which traditionally has been a complex task carried offline, is moving closer to real-time by harnessing HPC resources. Recently, several online DSA tools have been proposed in the literature. Their main feature is the collection of Wide-Area Measurement Systems (WAMS) using phasor measurement units (PMUs) for system monitoring purpose. The majority of these applications focus on real-time data analysis for detecting anomalies such as oscillatory modes, power imbalances and tripping events [27]. An online DSA platform has been deployed in the PJM control center, able to process 3000 contingencies every 15 minutes and calculate 16 interface stability limits across a system of 13,500 buses and 2,500 generators [5]. A distributed computing platform developed by EPRI and eMIT is capable of simulating 1000 contingencies for a test system of 20,502 buses and 3,263 generators in under 27

UK. G. Jamgotchian and P. Panciatici are with RTE, France. P. Duchesne is with Pepite, Belgium. S. Cole and C. Merckx are with Tractebel, Belgium. 
minutes [6]. Another recent example is a transient stability platform simulating 4,096 contingencies across 6 pre-fault operating points on a 25,355-bus system at an average of 200 seconds [7].

These implementations are able to analyze the central operating scenario in real time using high-fidelity simulations. However, one aspect that still remains elusive is the integration of operational-time uncertainties in the DSA process; the very high density of analysis required to guarantee statisticallysignificant exploration of the uncertainty space renders realtime Monte Carlo DSA frameworks beyond reach. One way to tackle this issue is to carry out a large part of the required computations offline. In other words, the operator can anticipate in advance the range of operating points likely to occur in the near future and thus carry out some analysis beforehand that will subsequently be used to support real-time decision-making. In this approach, time-domain simulations and machine learning techniques, such as Artificial Neural Networks [23] and decision trees (DTs) [24], can be combined to construct predictors of dynamic stability [26]. By keeping this process outside real-time constraints, predictors can be trained on a very large number of dynamic simulations to infer the system's stability boundary. The resulting predictors can subsequently be deployed within the online monitoring platform for real-time decision support [25]. For example, researchers in [11] utilize dynamic simulations to train a DT for monitoring a specific contingency in the French network. In a similar vein, authors in [12] propose a DT-based approach for online DSA and preventive control. Two DTs are generated on a daily basis; one for detecting security issues on the basis of observable variables and one for providing online decision support for suitable preventive measures. The platform is tested on a 400-bus model of the western Danish power system. Despite promising results, existing implementations lack the scalability required for practical DSA of large systems in combination with large numbers of contingencies.

The iTesla project [20] has developed a prototype toolbox for DSA of large-scale grids that combines offline and online functionality. The high-level concept of iTesla is to carry out a large number of dynamic simulations offline so as to identify a system's stability boundary and subsequently distill the results in security rules usable in real-time, essentially creating proxies for the full security assessment problem [3]. Using information computed offline to inform online operation has been applied in the past (e.g. [28]) but the iTesla platform implements this idea at an unprecedented scale, paving the way for DSA at the pan-European level. We indicatively mention that analyzing post-fault stability of 5000 operating points across a list of 1000 contingencies (France alone has almost 2000 credible contingencies) requires 5 million dynamic simulations. The offline proxy generation process will be carried out on a regular basis (e.g. weekly) to ensure security rules reflect the up-to-date underlying system reality.

This paper presents the iTesla offline DSA platform, describing its structure, implementation and a sample of the results obtained. The platform is based on a number of mature commercial tools, which are interfaced with custom code for interoperability and to provide specific functionality. In particular, we describe how the platform has been designed to efficiently leverage HPC resources in order carry out dynamic security analysis at an unprecedented scale. Results from a pilot case study on the French transmission network are presented and analyzed.

About 7,160 operating points have been generated to explore different demand and renewables production scenarios. Each of these operating points has been simulated against a list of 1980 contingencies, resulting in a total of over 14 million dynamic simulations. For each simulation, eleven indices were computed to characterize post-fault system stability, such as transient stability, voltage etc. All this information was subsequently condensed with the aid of machine learning using DTs. Two DTs of different complexity were trained for each combination of contingency and post-fault-index; over 40 thousand DTs were trained in total. In this paper we demonstrate that DSA and machine learning are tasks highly suited for HPC with almostperfect performance scaling, while also highlighting challenges likely to be encountered in such workflows.

\section{DSA FOR LARGE-SCALE SYSTEMS}

The iTesla DSA platform consists of two main components; an online and an offline module. The offline module is to be executed regularly (e.g. on a weekly basis) to reflect expected system conditions. It aims at exploring the network state-space to infer the separation between stable and unstable states using data mining techniques and high-fidelity dynamic simulations. More precisely, in the iTesla offline module, the system stability boundary is sampled using Monte Carlo techniques to generate a large number of realistic operating points, similar to what has been observed historically. Each of these operating points can then be analyzed in detail, to assess their robustness against a list of credible contingencies. Subsequently, machine learning techniques are used to compress the outcomes of the simulations into a set of rules describing the stability domain in terms of pre-fault state variables. Provided that the initial sampling density is sufficient, the above method can infer the underlying security boundary for each contingency and produce accurate proxies for pre-fault classification. The online workflow operates close to real-time, and is therefore limited in computational resources. The offline-generated security rules are used to identify contingencies that require more careful analysis in the online time frame. In this paper we focus on the offline sampling and rule generation workflow, which is detailed below.

\section{A. Stochastic sampling}

The first step to identifying relevant operating states is to sample the stochastic variables that are outside of the TSO's control such as loads and injections of intermittent sources. The aim is to construct a statistical model that approximates the multivariate probability distribution of all stochastic variables on the basis of large historical datasets of on-site measurements. There are several challenges to building such a multivariate model due to the high data dimensionality, the complexity of marginal distributions as well as the non-linearities of the dependence structure. To this end, a novel approach based on copulas, dimension reduction and clustering techniques has 
been developed in iTesla (more information in [13]). The model serves as the engine that generates the thousands of scenarios required for the Monte Carlo approximation of the system stability boundary. The statistical model is trained on a portion of the historical dataset deemed relevant with respect to system conditions such as weather and electrical demand.

\section{B. Simulation initialization}

The second step is to build a complete system state for each sampled scenario by inferring the corresponding market conditions and TSO control actions such as generation dispatch and system topology setup. Data-mining on historical data in conjunction with sophisticated optimization techniques are employed to infer a likely network state and ensure consistency with past operation of the system. This process consists of three distinct steps.

\section{1) Topology initialization}

The first phase is inferring the system topology. In this particular case, topology refers to the state description of all substations with more than one electrical node; this can have a profound impact on power flows throughout the network. A novel technique based on employing DTs trained on historical data to predict the most likely system topology, while also considering inter-variable correlations, has been developed.

\section{2) Starting point initialization}

Having defined the system topology, the next step is the inference of all other control variables such as real and reactive power dispatch, tap changer positions, voltage levels and power flows. In the interest of ensuring consistency with past system experience, chance constraints are introduced to bound operational decisions' probabilities according to observed data. However, the most important complication of this step is the computational burden imposed by the optimization problem; AC Optimal Power Flow (ACOPF) is a notoriously hard Mixed-Integer Non-Linear Problem (MINLP). These computational challenges have been addressed by reformulating the MINLP into a Mathematical Program with Equilibrium Constraints (MPEC) as well as applying an algorithm based on progressive filtering and a gradual introduction of constraints. The solution strategy is explained in more detail in [14].

\section{3) Load flow and stabilization}

After completing the initialization procedure, all unit commitment and topology control decisions are fixed and an AC load flow is performed to validate feasibility of the optimization problem where all constraints are enforced concurrently. Finally, another AC load flow is performed, this time using the dynamic simulator engine in order to convert the initial condition to the native data format of the EUROSTAG dynamic solver.

\section{Impact assessment}

The third and most resource-intensive task of the DSA workflow is the execution of a vast number of post-fault dynamic simulations. Each initialized system snapshots is combined with a predefined list of credible contingencies, and a time-domain simulation is executed for all combinations. The impact of a contingency on a pre-fault snapshot is summarized in a number of post-fault indices that have been developed to detect steady-state and dynamic phenomena such as over/under-voltage, angular and small signal instability. For more information, the interested reader is pointed to the relevant sources [21] and [22].

\section{Machine learning}

Finally, for each contingency and post-fault indicator, a DT is trained on the corresponding pre-fault state variables and postfault results to generate a set of security rules suitable for (near) real-time use in the online system. The performance of the DTs, measured in terms of the misclassification error on unseen datasets, can be tuned using a plethora of user-defined parameters related to the DT training process, such as depth and leaf purity. Furthermore, given that any pre-fault state variable is a potential classifying variable, a variable selection step precedes the training process in order to identify variables which are likely to have classifying power, considerably reducing training times. In the iTesla platform, two distinct types of DTs are trained for each contingency and post-fault indicator; a 'reduced' and a 'full' tree. Full DTs are trained on the full set of state variables. On the other hand, reduced DTs are trained solely on real power injections. The 'full' DTs are used in online contingency filtering, while 'reduced' DTs are embedded in a linear optimization problem to delineate the stable pre-fault system boundaries and determine whether the system is recoverable through corrective actions. More information on the attributes of the different DTs is provided in section IV.D.

\section{High PeRformance COMPUTING IMPLEMENTATION}

A typical large grid consists of several thousand buses and lines, with thousands of credible contingencies. Combining this with the thousands of possible operating conditions that are necessary to capture different demand scenarios, output of intermittent renewable sources etc., requires millions of dynamic simulations to analyze each combination, with typical computation times in the order of 1 minute per simulation. As a result, the computational load associated with dynamic simulations is in the order of millions of core-hours. The same holds true for DT training, where computation times for binary classification across tens of thousands of variables can exceed ten minutes. Given that offline rules should be updated on a regular basis, to account for changes in customer and market behavior and in the physical system itself, it is clear that the performance achieved by a straightforward sequential platform implementation is not viable. However, given the independence between contingencies, there is significant scope for parallelization to render large-scale DSA a tractable task. In this section we discuss the HPC implementation of iTesla platform in detail.

\section{A. Hardware setup and Platform Implementation}

In recognition of iTesla's significance in pioneering largescale DSA, the project was granted access to the petascale HPC facility Curie (CEA, France) [16] by the Partnership for Advanced Computing in Europe (PRACE) [4]. The offline module was run on Curie's thin nodes: 5,040 nodes equipped with two 8-core Intel Xeon E5-2680 CPUs (80,640 cores in total) and 64GB of RAM, running Linux CentOS 6 and communicating over an Infiniband QDR network. Under the access arrangements, up to 10,000 cores were used simultaneously in blocks of 24 hours (wall clock time). 
Figure 1 depicts the functional HPC implementation of the iTesla offline platform. A Master-Workers architecture is used, and task parallelism is implemented using a hybrid MPI/thread implementation. One node acts as the master and communicates with the 16 worker threads (one per CPU core) on each of the 624 worker nodes by means of the Message Passing Interface (MPI) protocol [15]. A single MPI task is spawned on each worker node at job start and it dispatches tasks to the node's 16 cores by creating threads. In Figure 1, unit tasks (programs or scripts) are indicated by dark grey blocks and MPI communication is indicated by solid arrows. The dark grey blocks are organized according to their functional units described in Section II. The different data storage sources required for each task are also shown. For example, the statistical model to be used for generating stochastic scenarios is loaded from central storage before commencing the batch sampling process. The computed DTs, the final output of the offline DSA workflow, are stored in a dedicated database for later use in order to support online operation.

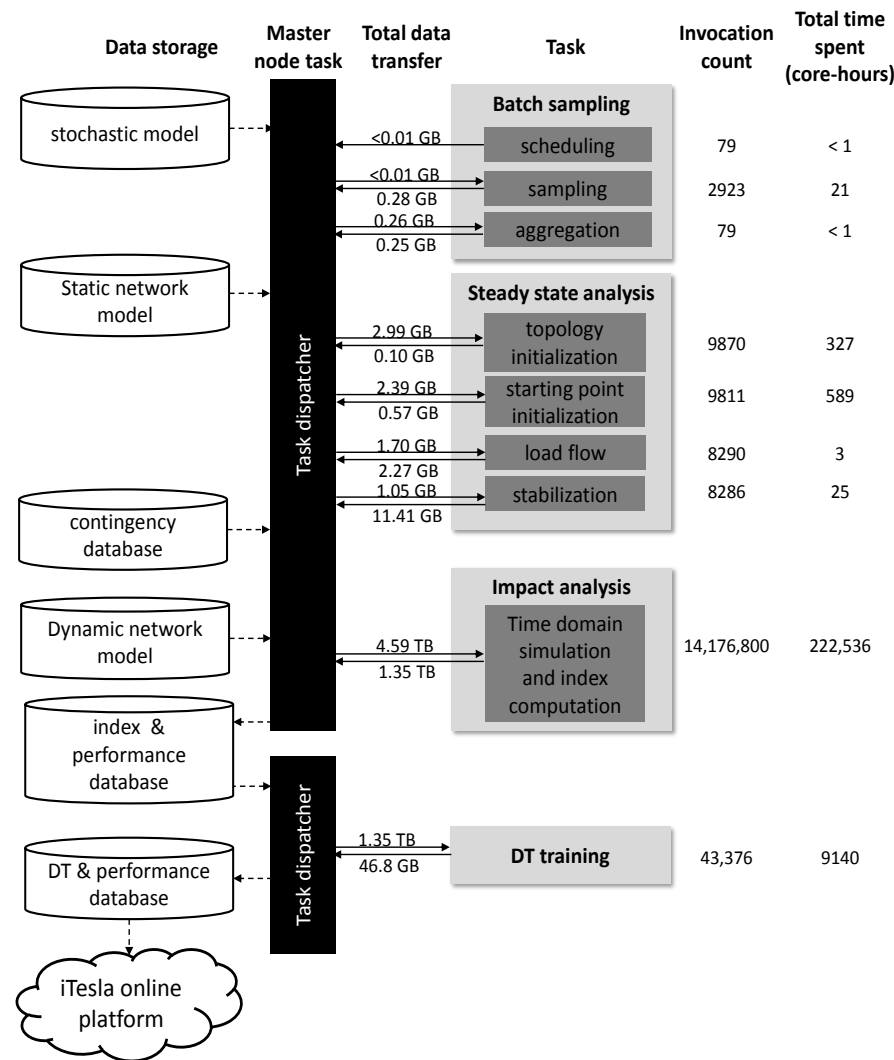

Fig. 1. HPC implementation of the iTesla offline platform. Invocation counts, aggregate data transfer volumes and computation times are shown for a 23 hour sampling run (top) and the associated decision tree training run (bottom; 9 hours wall clock time).

All tasks communicate through the master node for maximum scheduling flexibility: tasks are dispatched to the first available worker, ensuring continuous utilization of CPU resources. This is known as dynamic load balancing and is a fundamental requirement for efficient resource use in parallel programming environments. Dynamic load balancing is even more relevant when task execution times are unknown, as is the case with dynamic simulations where the time taken for the system to reach steady-state is highly dependent on the initial condition and the type of fault examined. In this particular application, the master node does not become a bottleneck due to the relatively long run times of each task, but this implementation aspect may need reconsideration for the orchestration of higher task rates, where the master node processing overhead becomes an issue.

The platform has been implemented as a highly modular architecture. The different modules (sampling, starting point initialization etc.) plug into the core platform, and interfaces between the core and each module have been designed to be generic and independent of any specific tool. A plugin design has also been chosen for data management to facilitate adaptation of the platform to different kinds of data storage. The master node platform was implemented in Java and C++, and the worker node platform was implemented solely in $\mathrm{C}++$. The platform has been released as open source for further community development [30].

\section{B. Module implementation}

The iTesla offline platform performs a sequence of complex operations to sample and analyze power system states. Rather than developing code from scratch, the platform incorporates a number of mature commercial tools (EUROSTAG, Knitro, Xpress, Matlab) and interfaces them with custom code in a variety of languages (java, C++, Matlab, AMPL).

The stochastic sampling module has been implemented in Matlab. It consists of three tasks that are run sequentially to generate a batch of sampled operating states. The sampler operates most efficiently in batch mode, taking advantage of the vectorization speed-up. Note that the parameterization of the statistical model may take place outside the HPC platform. Overall, there are two important considerations in its implementation. First of all, we want to avoid sampling more stochastic realizations than we can process given the target computational budget. In addition, since the stochastic model consists of several independent cluster-models of asymmetric complexity, we want to adopt a dynamic load balancing scheme and sample each model constituent individually. For these reasons, sampling functionality has been split in three distinct tasks to remove bottlenecks and enable continuous use of all available resources. The first task, named 'scheduling', schedules how many realizations will be sampled from each cluster-model; this stratification is critical to ensure representativeness even in small sample sizes. The second task, named 'Sampling', performs the actual sampling from each cluster-model. The third task, named 'Aggregation', consolidates all cluster samples to a merged sample set.

The steady state analysis module consists of four serial tasks. The 'topology initialization' task is first carried out. The optimization problem is implemented in AMPL and employs the Xpress solver. This task also makes use a topology prediction model implemented in Java, which has been previously executed outside the HPC platform. The optimizer decides on substation topologies while taking into account grid modeling considerations and any dependence with the sampled stochastic variables. The second task named 'starting point initialization' is responsible for setting up all control variables; it is implemented in AMPL and uses the Knitro solver. The third 'load flow' task re-executes the preceding step with the computed control decision variables as input for validation purposes. The final 'stabilization' task involves a steady-state 
simulation engine so as to ensure pre-fault system stability and initialize the time-domain simulation to be executed thereafter.

For the purposes of this study, EUROSTAG was used to carry out dynamic simulations [29]. EUROSTAG covers the full range of stability phenomena from transient to long-term stability. A variable step-size algorithm was used; when highly dynamic phenomena are simulated, the step-size is automatically reduced to achieve the desired accuracy. Once the transients have died out, the step size increases to large values, thus achieving great simulation speeds. AMD ordering or the KLU linear solver can be selected for the solution of the linear system within the integration algorithm, depending on the size of the system to be simulated. For the French network, AMD ordering suffices, while for the European network the KLU solver is required. For the purpose of this project, EUROSTAG has been enhanced to improve its performance in HPC environments. Changes included faster initialization, removing the GUI and extending the API. The API is used to calculate stability indices and stop the simulation once a diagnostic is available. For the post-fault impact assessment, eleven indices have been implemented in Matlab and integrated with the simulation engine using a $\mathrm{C}$ interface.

Once the dynamic simulation and impact assessment tasks are complete, they result in a database filled with one record per sampled pre-fault condition. Each record contains all pre-fault steady-state variables and a symbolic value (stable/unstable) for each post-fault indicator and contingency. Security rules are then to be produced in the form of decision trees; one tree is created for each contingency-indicator pair. Note that the DT training process also involves a data cleaning stage, where each state variable is scanned for missing values; state variables with too many missing values are excluded from the training. As each DT can be trained independently, the training process can be efficiently parallelized and dispatched to an HPC platform. To this end, the input data set is prepared and packaged as a memory mapped file that is dispatched to each node of the HPC platform. The use of a memory mapped file reduces the CPU and memory footprint on each processing node, thereby limiting the performance needs strictly to the DT training algorithm. Each node produces a DT for which a text file representation is committed to a dedicated centralized database upon process termination.

\section{RESULTS}

Extensive pilot studies have been carried out on the French transmission network to evaluate platform performance. The French transmission network is a large system that consists of 1,955 transmission lines, 798 transformers, 1,886 buses, 411 generators and 127 shunt elements. The security assessment is performed on a list of 1,980 contingencies deemed credible by the French TSO. This list consists of 1,827 single line outages (simulated as a short circuit at middle of the line), 8 double line outages and 145 generator outages (simulated as a short circuit at the generator bus).

There were two separate HPC runs on the PRACE facility. The first was a sampling and analysis run on 10,000 cores to perform the dynamic simulations and indicator computations. Tasks were started for 23 hours (wall clock time) and the final task finished 3 minutes after the start of the final task. The second run, carried out afterwards, was run on 1,000 cores to train and test all decision trees. This process took a little over 9 hours to complete. For each run, the simulation outcomes are stored in two databases. The first is the results database. For the first run this contains the parameters describing each initial condition, and the resulting security indices for each contingency. For the second run, the results database stores the computed decision trees. The second database, the performance database, stores data on all tasks executed on the HPC platform (task descriptors, timings, message sizes, task exit codes etc.) for performance profiling. In this section the performance data are described in detail, and the power system analysis results are summarized.

\section{A. Performance results - Scaling performance}

In view of the huge computational load imposed by contingency simulation, the importance of adopting an efficient load balancing scheme is very clear. To this end, the effectiveness of the implemented scheme was first verified through scalability studies that showed near-perfect scaling with the number of cores employed, due to the embarrassingly parallel nature of the problem. Table I illustrates the application's scaling performance for five different runs using 512, 1024, 4096 and 10000 cores. The speedup is computed by dividing the number of dynamic simulations performed in one hour (wall clock time) by the number of cores. This confirms the previously known result [10] that dynamic computation load balancing for parallel contingency screening results in exceptional speedup performance.

TABLE I

SCALING PERFORMANCE

\begin{tabular}{ccc}
\hline Cores & Simulation executions & $\begin{array}{c}\text { Speedup factor } \\
\text { (relative to 512 cores) }\end{array}$ \\
\hline 512 & 5,0400 & 1 \\
1024 & 107,000 & 2.12 \\
2048 & 214,400 & 4.25 \\
4096 & 433,600 & 8.60 \\
10000 & $1,044,472$ & 20.72 \\
\hline
\end{tabular}

\section{B. Performance results - Dynamic simulations}

The total computation time occupied by the various offline platform tasks was equal to 223,500 core-hours, while 3,238 core-hours were spent in data transfers between modules. These numbers suggest that $98.52 \%$ of the available computation resources $(624$ nodes*16 worker threads*23.05 hours $=$ 230,131 core-hours) were used. This highlights the success of the dynamic load balancing scheme which limited idle time to just $1.48 \%$. The majority of this overhead (approx. 1\%) was incurred during the startup phase. In Figure 2 we plot the use of computational resources as a function of time, to obtain a chronological view of the experiment execution. For the sake of clarity, we show only the first half hour of HPC use.

As can be seen in Figure 2, the very first task being run is a load flow in order to build a consistent base case including stepup transformers. The base case contains the grid structure used in all offline modules. Subsequently, the stochastic sampling processes are launched followed by starting point initialization procedures. Overall, idle time is concentrated primarily in the first few minutes of the HPC run, when starting points are still being built and the stack of dynamic simulations has not yet been populated. At around the 900 second mark, when the first 
few complete starting points have been built, resource utilization rises to $100 \%$ due to the very large number of simulations to be executed. Periodically, the task scheduler preemptively orders the generation of new samples (in batches of 128) to keep computational load consistently close to $100 \%$. This first occurs near the 1600 second mark.

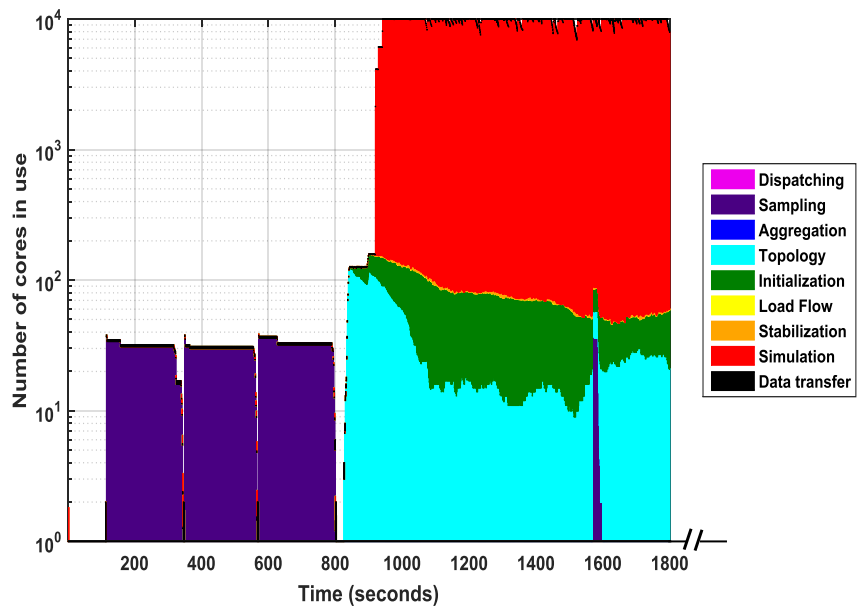

Fig. 2. Use of HPC resources as a function of time across different processes; logarithmic scale has been used to render tasks that occupy little time visible. Note that the figure focuses on the first 30 minutes (1800 seconds) of the total runtime of over 23.05 hours wall clock time ( $\sim 2,800$ seconds).

A summary of invocation counts, average computation times and data transfers per individual platform module are shown in Table II. Note that CPU time is split between the time spent performing the ascribed task and time expended on input/output (I/O) transfers. Data transfers are divided into input, output and temporary locally generated files.

TABLE II

INVOCATION COUNTS, MEAN COMPUTATION TIMES AND DATA TRANSFERS

\begin{tabular}{|l|r|r|c|c|r|c|}
\hline \multirow{2}{*}{ Module } & \multirow{2}{*}{ Instances } & \multicolumn{2}{|c|}{ CPU time (sec) } & \multicolumn{3}{|c|}{ Data transfer (MB) } \\
\cline { 3 - 7 } & & \multicolumn{1}{|c|}{ Task } & I/O & In & $\begin{array}{c}\text { Local } \\
\text { disk }\end{array}$ & Out \\
\hline Dispatching & 79 & 2.51 & 0.30 & 0.00 & 0.00 & 0.01 \\
\hline Sampling & 2,923 & 25.25 & 0.17 & 0.00 & 1.29 & 0.10 \\
\hline Aggregation & 79 & 3.38 & 0.27 & 3.36 & 6.67 & 3.29 \\
\hline Topology & 9,870 & 119.23 & 0.23 & 0.31 & 2.86 & 0.01 \\
\hline Initialization & 9,811 & 216.16 & 0.24 & 0.25 & 4.63 & 0.06 \\
\hline Load flow & 8,290 & 1.10 & 0.19 & 0.21 & 4.21 & 0.28 \\
\hline Stabilization & 8,286 & 10.65 & 0.24 & 0.13 & 39.93 & 1.41 \\
\hline Simulation & $14,176,800$ & 56.51 & 0.82 & 0.34 & 169.35 & 0.10 \\
\hline
\end{tabular}

The French system was modeled using 3,808 TSO-external stochastic variables; real and reactive power demand across all load buses (3,716 variables), real and reactive power across all cross-border lines (64 variables) and real power and voltage for all intermittent generation plants (28 variables). A statistical model consisting of 37 clusters has been parameterized on 2,976 historical observations (15-minute measurements from January 2013). Each invocation of the iTesla dispatching module schedules the generation of a batch of 128 samples. Subsequently, an instance of the sampling algorithm is launched for each cluster-model to generate the stochastic realizations. Dispatching and aggregating have very low computational cost, while the sampling process itself takes up 20 core-hours. Throughout the experiment duration a total of 10,112 random operating states are generated, but due to the limited run time of 23 hours only 9,870 samples pass to the topology inference step.

The four subsequent tasks aim at building realistic starting points for the dynamic simulation. Topology inference and system initialization are resource-intensive tasks while load flow and stabilization are negligible in comparison. Each module is launched once per sample, the total CPU time spent on all four tasks is 945 core-hours, accounting for less than $0.5 \%$ of the total computational budget. Figure 3 shows the distribution of computation times for the four different modules. The topology module uses DTs to forecast a likely system topology for each sample. This is a resource-expensive step with an average computation time of 2 minutes per module invocation; 59 realizations timed out. Initialization has the largest execution time variability, ranging from less than 30 to more than 600 seconds. Also note that during this step, a significant number of samples is discarded due to the userdefined process time-out of 1- minutes, as indicated by the spike at the 600 -second mark.

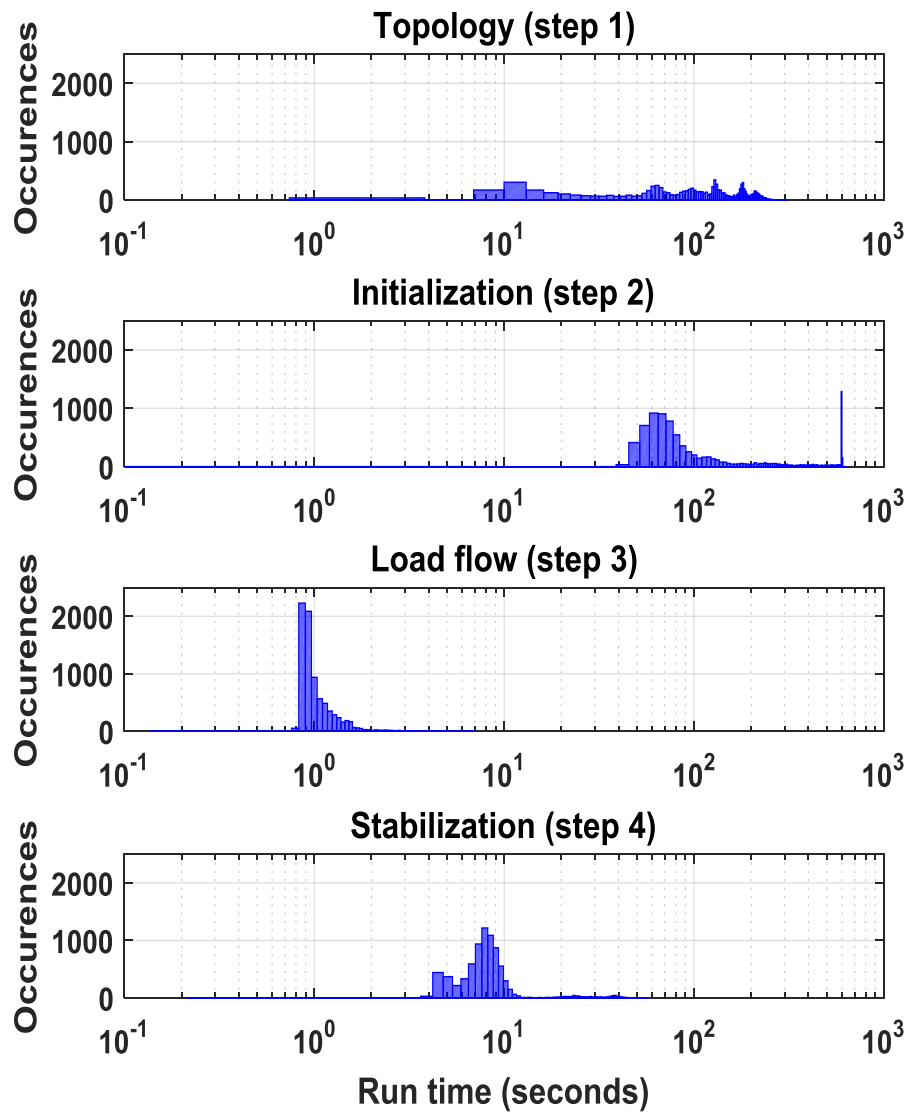

Fig. 3. Run time histograms for the four steps of steady-state analysis.

The execution of dynamic simulations and post-fault impact assessment constitute the most CPU-intensive tasks; more than $99.5 \%$ of the computational budget is expended on them. The average time to perform a single simulation and compute all 11 indices is 56.51 seconds; this is due to the large size of the system being analyzed which involves 34,368 state variables. The distribution of execution times of 14.2 million time domain simulations and the corresponding indicator computations are shown in Figure 4 (note the logarithmic frequency scale). 


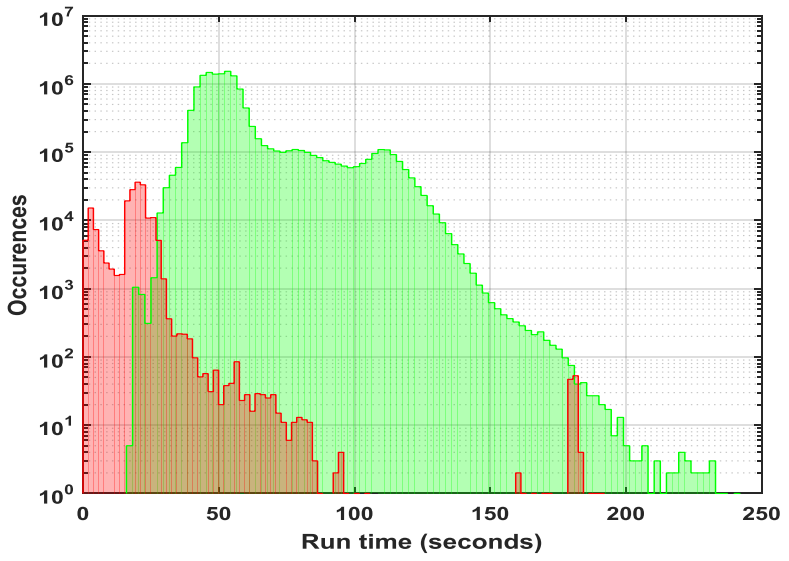

Fig. 4. Distribution of execution times of dynamic simulations. Green: successful runs; red: unsuccessful runs.

As can be seen above, simulation and impact analysis times occupy a diverse time range; from a few seconds up to more than 3 minutes. Unsuccessful runs are concentrated towards the lower end of the spectrum with the mean CPU time being 17.8 seconds, whereas successful runs have mean execution time of 57 seconds. Note that a simulation timeout of 180 seconds was imposed to ensure that problematic simulations do not unduly occupy CPU resources. This is evidenced by the cluster of unsuccessful runs at the 180 second mark. Some successful runs take more than that due to the subsequent impact analysis phase.

To further investigate the unsuccessful dynamic simulations, in Figure 5 we present two plots showing the percentage of unsuccessful simulations as function of the contingency index (panel a), and the operating point index (panel b). From the leftmost panel, it is evident that there are specific contingencies that regularly result in simulation failure. Some contingencies result in as high as $80 \%$ failure rates, meaning that more than 5700 out of the total 7160 simulations performed for that particular contingency fail to converge (including system collapse). Note that the cluster of contingencies that exhibit very high failure rates (around the 1800 index mark) correspond to double circuit outages. On the other hand, when examining the rightmost panel there are no specific operating points that consistently result in unsuccessful simulations. The undertaken analysis indicates that extra attention should be taken to identify contingencies that may consistently result in simulation failure. Subsequent analysis is required to understand and mitigate high numbers of unsuccessful simulations.
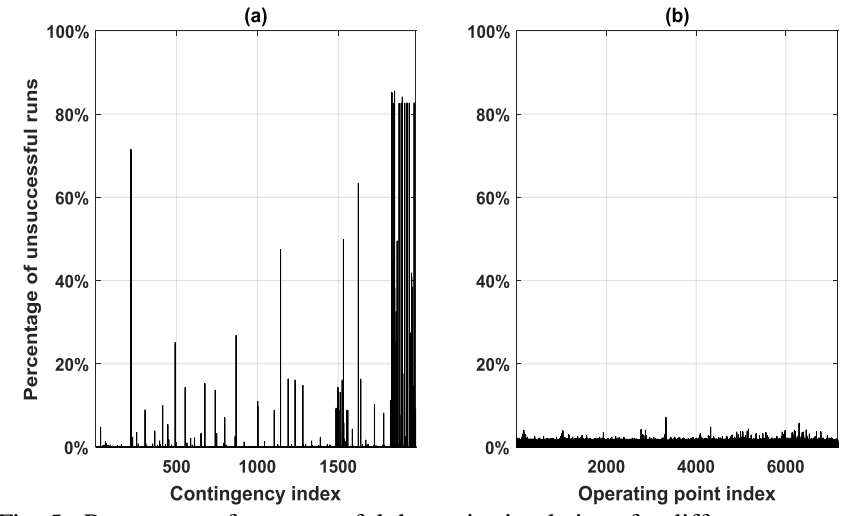

Fig. 5. Percentage of unsuccessful dynamic simulations for different contingencies (panel a) and different operating points (panel b).
As dynamic simulations place the largest burden on the HPC system, we consider the data volumes required. As discussed extensively in [7], time spent in I/O is one of the most severe bottlenecks of parallel contingency processing. In our approach, data transfers have been minimized by dispatching post-fault dynamic simulation and computation of stability indices as a single task that takes place in the same node. This is evident in the fact that whereas a large temporary file of average size 169.35 MB is generated during simulation containing thousands of signal traces spanning several seconds, the output file's average size is just $0.10 \mathrm{MB}$; it contains solely the indicator results to be fed to the machine learning algorithm. Whereas 2.2 petabytes of data were generated by the 14.1 million dynamic simulation runs, only 1.35 terabytes were relayed back to the master node.

\section{Performance results - Decision Tree training}

For the machine learning part, a second HPC run was scheduled to run on 127 worker nodes, each with 8 cores $(1,016$ cores in total). Given that the training of each DT can be carried out independently, a parallel implementation has been adopted. As before, dynamic load balancing is critical given the large range of execution times across DTs. Individual DTs were computed for each contingency in combination with each of a total of 11 post-contingency indices, covering a wide range of static and dynamic phenomena such as line overloads, over/under-voltages, small-signal stability, transient stability, loss of synchronization etc. In addition, as mentioned earlier, two types of trees are created; reduced DTs trained on 7,897 attributes and enhanced DTs trained on 25,378 attributes.

Overall, the total computation effort for training 43,376 trees $(1980 \times 11 \times 2$ minus 184 DTs that failed to converge) was 9,140 core-hours, corresponding to almost 9 hours wall clock time. For each DT, $80 \%$ of the 7,160 input-response pairs are randomly chosen and used for training, while the remaining 1,432 records are used for testing. It is worth noting that during each training instance, a $2.14 \mathrm{~GB}$ local file is generated. However, the average size of the final output files are only 0.54 MB and 1.67 MB for reduced and full trees respectively, with little variation across contingencies and indicator types.

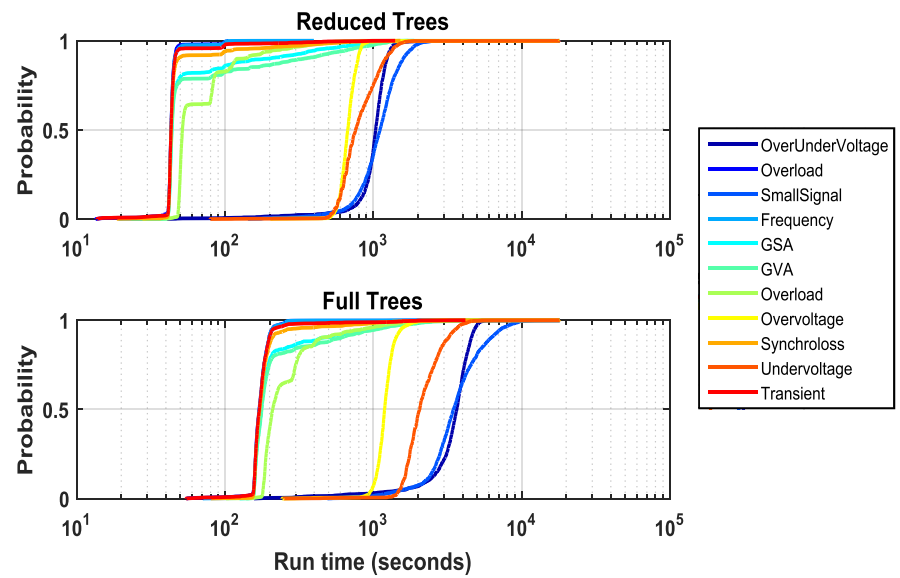

Fig. 6. Cumulative probability distribution of DT training execution times for reduced and full trees across eleven post-fault indices.

In Figure 6 we show the distribution of execution times for DT training on the different indices. The increased 
computational load involved in the training of full trees (bottom panel) is evident; the average execution time for full and reduced trees is 1124 and 391 seconds respectively. In addition, there are trees that take considerable amounts of time to be trained, some needing up to 5 hours. For example, full DTs trained on the small signal and over/under-voltage have average execution times of 3,871 and 3,536 seconds respectively. Overload is the indicator resulting in the smallest average training time of 195 seconds.

\section{Dynamic Security Assessment outcomes}

We proceed from the analysis of performance data to a summary of simulation results. In the present analysis we focus on four particular indices which have been extensively validated; line overload (OV), loss of synchronization (SL), generator speed automaton (GSA) and generator voltage automaton (GVA). GSA is an index signifying whether at least one generator was disconnected during the simulation due to an over- or under-frequency event; the corresponding frequency thresholds were set at $47.5 \mathrm{~Hz}$ and $55 \mathrm{~Hz}$ respectively. GVA is an index signifying that at least one generator was disconnected during the simulation due to an over- or under-voltage event; the corresponding voltage thresholds were set at 1.2 p.u. and 0.7 p.u. respectively.

The indicator results of 14 million dynamic simulations are displayed in Figure 7, where we show the percentage of postfault unstable operating points per contingency across the four different indicators. Note that the contingencies are sorted (starting from those that result in post-fault instability in a large number of samples) for each individual post-fault indicator. Focusing on the OV, SL and GSA indicators, only a few hundred contingencies result in post-fault instability with a probability larger than $1 \%$. This is as expected, given that the French network should be robust against most disturbances, but a few sampled extreme conditions can lead it to post-fault instability. Regarding the GVA indicator, more than half of the contingencies analyzed result in post-fault problems with a probability greater than $10 \%$ due the chosen automaton configuration being highly sensitive to voltage excursions.

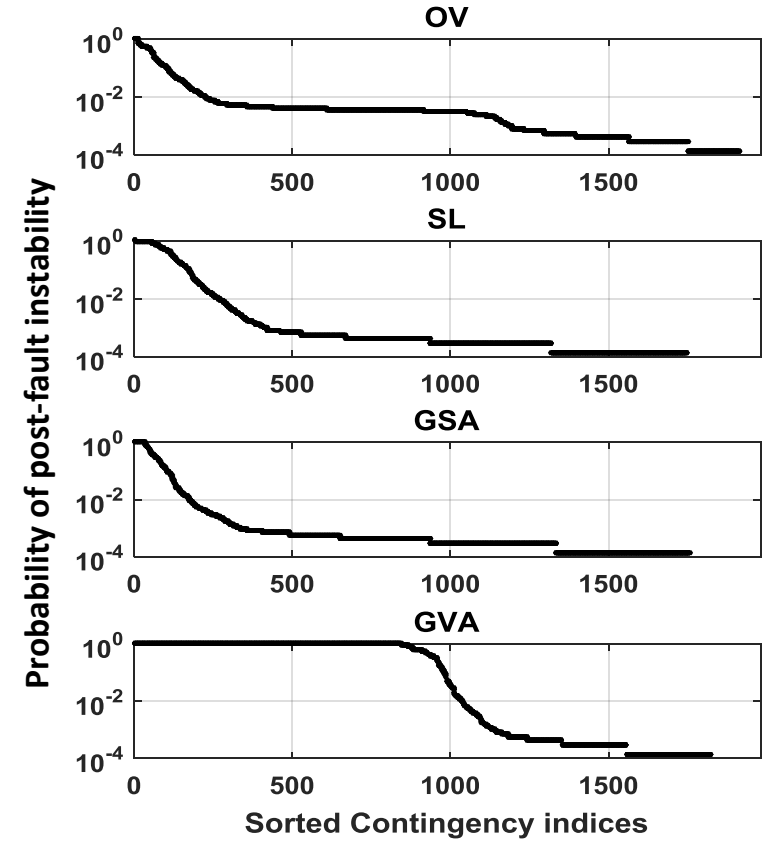

Fig. 7. Probability of post-fault instability for the 1980 examined contingencies according to the four post-fault indicators.

Moving on to the machine learning results, the main metric of interest is the test error associated with each decision tree. In addition, tree sizes are useful in communicating the complexity of the resulting classifier. Average metrics are shown in Table III, while the distributions of test errors are shown in Figure 8. As can be seen, all eight DT classes have good predictive power. As expected, full trees perform better overall and are more compact, due to their access to more classifying variables.

TABLE III

AVERAGE TEST ERRORS AND TREE SIZES

\begin{tabular}{lcccc} 
& OV & SL & GSA & GVA \\
\hline \cline { 2 - 5 } Reduced Trees & & & & \\
\hline Test error & $0.24 \%$ & $0.12 \%$ & $0.40 \%$ & $0.48 \%$ \\
Tree size & 3.34 & 1.81 & 4.05 & 3.59 \\
\hline Full Trees & & & & \\
\hline Test error & $0.22 \%$ & $0.04 \%$ & $0.17 \%$ & $0.23 \%$ \\
Tree size & 3.41 & 1.34 & 2.6 & 2.93 \\
\hline
\end{tabular}

Despite the fact that the average test errors in all DT types are below $0.5 \%$, it is important to note that, as evidenced in the distribution of errors (shown in Figure 8), some particular DTs have test errors above $10 \%$. Typically, such an error rate would be unacceptable for a highly sensitive task such as DSA; missed alarms can entail significant consequences for the system. Given the operator's asymmetric view on missed and false alarms, it may be prudent to modify the DT training algorithm by penalizing missed alarm events and thus biasing DT training towards detecting binding events at the expense of raising more false alarms. In the presented case study such re-weighting was not implemented. Beyond optimizing the DT training process for the application at hand, it is also important to define novel methods that can ascribe a validity index to a particular prediction. For example, the predictive capability of a DT is different when the operating point being analyzed is very close to the population already encountered in the training set versus cases where it is fundamentally different to the alreadyencountered population. Alternative analysis routes should be followed when the DT is deemed insufficiently trained or trained on an irrelevant pre-fault state-space domain. 


\section{Reduced Trees}
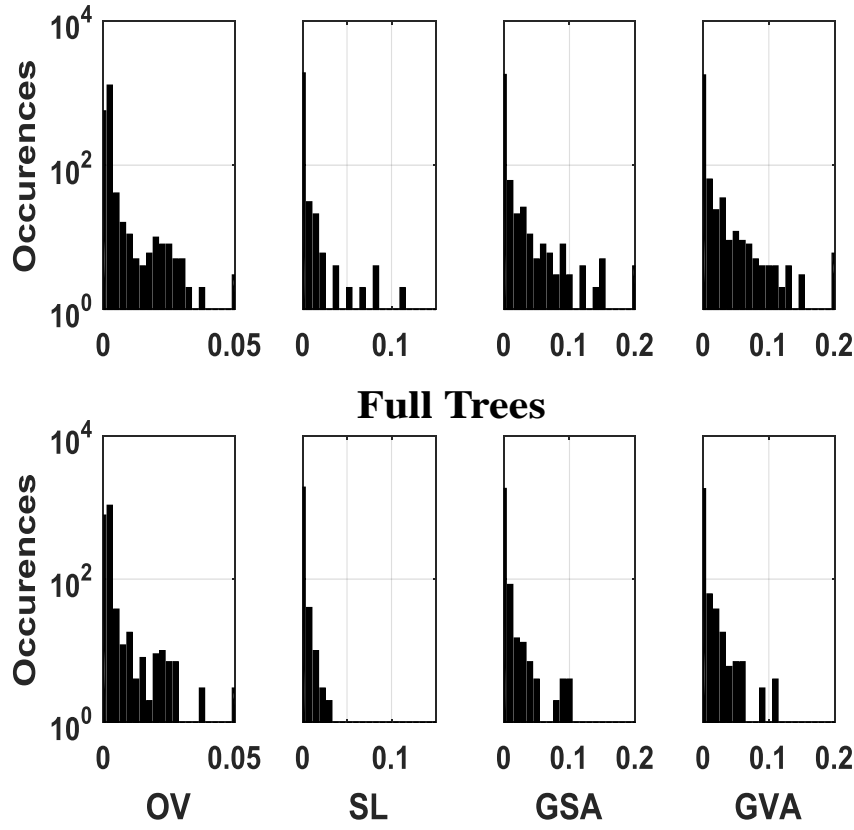

Fig. 8. Histogram of test errors for four indices in reduced and full trees.

\section{CONCLUSION}

In this paper HPC has been used to implement a massively parallel DSA platform. The constituent parts of the iTesla offline platform have been presented along with details of the HPC implementation and results of a case study on the French transmission grid carried out on 10,000 parallel cores. Extensive performance profiling has been carried out to assess scalability potential and identify any computational bottlenecks. The excellent performance scaling underscores the suitability of HPC for large-scale DSA analysis. Furthermore, the relatively modest communication requirements and embarrassingly parallel nature of the problem means that it may also be considered in a High Throughput Computing (HTC) environment, for example on distributed compute clouds. Finally, the demonstrated classification accuracy suggests that the DTs can meaningfully assist decision making in the (near) real-time domain.

In ongoing work, the core iTesla platform as presented here, is being enhanced to ensure the end product can carry-out panEuropean DSA in the most efficient manner. To this end, a novel importance sampling scheme is being developed to ensure HPC resources are spent on simulations likely to provide information gain to the classification problem at hand. In addition, the platform is being extended to support alternative simulation engines to ensure platform inter-operability among European TSOs. The online-offline DSA architecture stands to gain tremendously from large-scale experiments that will formally investigate the required updating frequency of the offline security rules. In a similar vein, it will be constructive to further investigate the amount of samples necessary for carrying out a comprehensive system state-space exploration that leads to adequate predictive capability.

\section{ACKNOWLEDGEMENT}

We gratefully acknowledge PRACE for awarding us access to resource CURIE based in France at Très Grand Centre de Calcul. The authors would also like to thank all iTesla partners for their contribution to platform development.

\section{REFERENCES}

[1] P. Panciatici, G. Bareux and L. Wehenkel, "Operating in the Fog", IEEE Power and Energy Magazine, vol. 10, no. 5, pp. 40-49, Sept. 2012.

[2] J.W. Bialek, "Why has it happened again? Comparison between the UCTE blackout in 2006 and the blackouts of 2003", IEEE PowerTech, Lausanne, Jul. 2007.

[3] V. Krishnan, J.D. McCalley, S. Henry and S. Issad, "Efficient database generation for decision tree based power system security assessment", IEEE Transactions on Power Systems, vol. 24, no. 4, pp. 2319-2327, Nov. 2011.

[4] PRACE Research Infrastructure website, http://www.prace-ri.eu.

[5] Jianzhong Tong and Lei Wang, "Design of a DSA Tool for Real Time System Operation", Powercon 2006, Chongqing, China, Oct. 2006.

[6] R. Schainker, G. Zhang and P. Hirsch, "On-line dynamic stability analysis using distributed computing", IEEE PES GM '08, pp. 1-7, Pittsburgh, 2008.

[7] S. Smith, C. Woodward, Liang Min, Chaoyang Jing and A. Del Rosso, "On-line transient stability analysis using high performance computing", IEEE PES ISGT 2014, pp. 1-5, Washington, Feb. 2014.

[8] D.M. Falcao, C.L.T. Borges an G.N. Taranto, "High Performance Computing in Electrical Energy Systems Applications", High Performance Computing in Power and Energy Systems, Spring, 2013.

[9] ISO New England, 2013 Regional Electricity Outlook, 2013.

[10] Z. Huang, Y. Chen and J. Nieplocha, "Massive contingency analysis with high performance computing", IEEE PES GM '09, pp. 1-8, Calgary, 2009.

[11] V. Krishnan, J.D. McCalley, S. Henry and S. Issad, "Efficient Database Generation for Decision Tree based Power System Security Assessment", IEEE Transactions on Power Systems, vol. 26, no. 4, pp. 2319-2327, Nov. 2011.

[12] C. Liu, K. Sun, Z.H. Rather, Z. Chen, C.L. Bak, P. Thøgersen and P. Lund, "A Systematic Approach for Dynamic Security Assessment and the Corresponding Preventive Control Scheme Based on Decision Trees", IEEE Transactions on Power Systems, vol. 29, no. 2, Mar. 2014.

[13] M. Sun, I. Konstantelos, S. Tindemans and G. Strbac, "Evaluating Composite Approaches to Modelling High-Dimensional Stochastic Variables in Power Systems," Power Systems Computation Conference (PSCC), Genoa, 2016.

[14] M. Ruiz, J. Maeght, A. Marié, P. Panciatici and A. Renaud, "A progressive method to solve large-scale AC Optimal Power Flow with discrete variables and control of the feasibility", Power Systems Computation Conference (PSCC), Wroclaw, 2014.

[15] W. Gropp, E. Lusk, N. Doss and A. Skjellum, "A high-performance, portable implementation of the MPI message parsing interface standard", Parallel computing, vol. 6, no. 2, pp. 789-828, 1996.

[16] Curie supercomputer. http://www-hpc.cea.fr/en/complexe/tgcc-curie.htm

[17] Z. Huang, Y. Chen and D. Chavarria-Miranda, "High-Performance computing for Real-Time Grid Analysis and Operation", High Performance Computing in Power and Energy Systems, Spring, 2013.

[18] C.L.T Borges, D.M Falcao, J.C.O Mello and A.C.G. Melo, "Composite Reliability Evaluation by Sequential Monte Carlo Simulation on Parallel and Distributed Processing Environments", IEEE Transaction on Power Systems, vol. 16, no. 2, May 2001.

[19] Y. Chen, S. Jin, M. Rice and Z. Huang, "Parallel state estimation assessment with practical data", IEEE PES GM, Vancouver, 2013.

[20] iTesla project. http://www.itesla-project.eu/

[21] F.R.S. Sevilla and L. Vanfretti, "Static stability indexes for classification of power system time-domain simulations", IEEE PES ISGT 2015, pp. 15, Washington, 2015.

[22] L. Vanfretti and F.R.S Sevilla, "A three-layer severity index for power system voltage stability assessment using time-series from dynamic simulations", ISGT-Europe 2014, Istanbul, pp. 1-5, Oct. 2014.

[23] Q. Zhou, J. Davidson and A.A. Fouad, "Application of artificial neural networks in power system security and vulnerability assessment", IEEE Transactions on Power Systems, vol. 9, no. 1, pp. 525-532, Feb. 1994.

[24] K. Sun, S. Linkhate, V. Vittal, V. Sharma Kolluri and S. Mandal, "An online Dynamic Security Assessment Scheme Using Phasor 
Measurements and Decision Trees", IEEE Transaction on Power systems, vol. 22, no. 4, pp. 1935-1943, Nov. 2007.

[25] L. Wehenkel, T. Van Cutsem and M. Ribbens-Pavella, "An artificial intelligence framework for online transient stability assessment of power systems," IEEE Transactions on Power Systems, vol. 4, no. 2, pp. 789800, May 1989.

[26] L. Wehenkel, Automatic learning techniques in power systems, Springer, 2012.

[27] N. Kakimoto, M. Sugumi, T. Makino and K. Tomiyama, "Monitoring of Interarea Oscillation Mode by Synchronized Phasor Measurements", IEEE Transactions on Power Systems, vol. 21, no. 1, Feb. 2006.

[28] L. Loud, S. Guillon, G. Vanier, J.A. Huang, L. Riverin, D. Lefebvre and J.-C. Rizzi, "Hydro-Québec's challenges and experiences in on-line DSA applications," IEEE PES General Meeting 2010, Minneapolis, pp. 1-8, 2010.

[29] Eurostag software, http://www.eurostag.be

[30] iTesla Power System Tool repository, https://github.com/itesla/ipst 